\title{
Wandel und Variation der Genitivstellung in einem diachronen Predigten-Korpus
}

\author{
Eine epochenübergreifende Längsschnitt-Studie
}

\begin{abstract}
Die Forschung zur Diachronie der Stellung des adnominalen Genitivs im Deutschen ist ein gutes Beispiel dafür, dass sich einzelne Fallstudien nur schwer zu einem schlüssigen Gesamtbild kombinieren lassen, wenn diese jeweils Zeitabschnitte mit unterschiedlichen Textsorten aus verschiedenen Regionen untersuchen. Durch uneinheitliche Quellenlage und Methodik wird die Vergleichbarkeit von Einzelstudien stark eingeschränkt. In diesem Beitrag wird ein alternativer Ansatz verfolgt, der auf einen zeitlichen Längsschnitt setzt. Indem sich der Fokus auf eine bestimmte Textsorte richtet, wird die annähernde Abdeckung der Zeit vom 9. bis zum 19. Jahrhundert auf einer gleichförmigen, wenn auch nicht repräsentativen Grundlage möglich. Als Textsorte wurden hier Predigten gewählt, da sich diese als Prosatexte gut für die Untersuchung syntaktischer Fragestellungen eignen und eine relativ lange, breite und kontinuierliche Überlieferung aufweisen. Neben der zeitlichen Entwicklung wird die Auswirkung verschiedener interner und externer Faktoren auf die Genitivstellung untersucht. In der Diskussion der Ergebnisse zeigt sich einerseits, dass der nachgestellte Genitiv durch seine größere syntaktische Flexibilität begünstigt wird; zum anderen wird deutlich, dass der Schwund des Genitivs im Gesprochenen nicht ohne Folge für seine Stellung blieb.
\end{abstract}

Keywords: Genitiv, Syntax, Diachronie, Korpuslinguistik, Variation, Wandel

\section{Zur Diachronie der Genitivstellung}

Die Stellung des adnominalen Genitivs ist ein altes Thema in der historischen Syntax des Deutschen, das angefangen mit Harczyk (1898) über Wagner (1905), Behaghel (1930), Carr (1933), Ebert (1988), Oubouzar (1997), Lanouette (1998), Prell (2000), Demske (2001) bis hin zu Kopf (2018), um nur einige Arbeiten zu nennen, bereits seit über einem Jahrhundert immer wieder zu Untersuchungen anregt. Dabei hat bereits Behaghel (1930) eine Darstellung der Grundzüge der

Simon Pickl, Universität Salzburg, Fachbereich Germanistik, Erzabt-Klotz-Str. 1, 5020 Salzburg und University of Cambridge, Cambridge, CB3 9DA, E-Mail: simon.pickl@sbg.ac.at 
Entwicklung vorgelegt, die bis heute nicht wesentlich revidiert oder modifiziert worden ist:

Die Entwickelung in der Stellung des nichtpartitiven Genitivs vollzieht sich in drei Hauptstufen. Zunächst stehen Genitive jeder Art voran, wenn nicht das Gesetz der wachsenden Glieder ${ }^{\left[{ }^{1}\right]}$ Platz greift; sodann wandern die Sach- und Abstraktbezeichnungen hinter das regierende Substantiv, schließlich erfahren Personenbezeichnungen, die nicht Eigennamen sind, das gleiche Schicksal. (Behaghel 1930: 49-50)

Diese relative Chronologie ist seit annähernd neunzig Jahren nicht wesentlich überholt worden (vgl. für das Althochdeutsche jedoch Oubouzar 1997); lediglich über die genaue zeitliche Verortung der Vorgänge gehen die Einschätzungen auseinander. Üblicherweise wird die Gesamtentwicklung als eine kontinuierliche Zunahme der Nachstellung dargestellt, als „sukzessive Rechtsverlagerung“ (Ágel 2000: 1858), die sich „über Jahrhunderte hinweg“ (Kopf 2018: 86) zieht, wobei der „Zeitpunkt des Stellungswandels“ (Kopf 2018: 86) von Darstellung zu Darstellung unterschiedlich beurteilt wird. Unklarheit herrscht dabei auch über die quantitative Entwicklung der Nachstellung, da sich die Zahlen der verschiedenen Studien nur schwer zu einem Gesamtbild vereinigen lassen, sodass sich „eine diachrone Entwicklung [...] daran [...] nicht festmachen“ (Kopf 2018: 87) lässt. Dies liegt unter anderem daran, dass sich die Genitivstellung in Abhängigkeit von Behaghels Bezeichnungstypen unterschiedlich entwickelt; hinzu kommen Unterschiede in der Datenbasis und der Methodik verschiedener Studien. Kopf (2018: 87) führt aus, „dass eine direkte Vergleichbarkeit der Zahlen, insbesondere aufgrund der unterschiedlichen Korpuszusammenstellung, aber sicher auch bedingt durch unterschiedliche Klassifizierungsmethoden, nicht sinnvoll ist“.

Die Problematik der Korpuszusammenstellung sei nur an einigen wenigen Beispielen illustriert (Abb. 1): Behaghel (1930) und Fritze (1976) verwenden eine Vielzahl verschiedener Textsorten, die nicht gleichmäßig über die Zeit verteilt sind; Fritzes Korpus ist jedoch räumlich ausgewogen. Ebert (1988) untersucht Briefe von Nürnberger Bürgern aus dem 16. Jahrhunderts, während Barufkes (1995) Zahlen ausschließlich auf Verstexten basieren. Prell (2000) verwendet verschiedene Arten von Prosatexten aus dem gesamten hochdeutschen, Tükör (2008) Urkunden aus dem niederösterreichischen Raum. Demske (2001), Scott (2014) und Niehaus (2016) untersuchen Zeitungen: Demskes Zeitungen stammen aus Wolfenbüttel (1609) sowie Frankfurt und Straßburg (1667), während die Zeitungsquellen von Scott and Niehaus räumlich ausgewogen sind (und zum Teil übereinstimmen).

1 Das Gesetz der wachenden Glieder „,besagt, daß von zwei Gliedern, soweit möglich, das kürzere vorausgeht, das längere nachsteht“ (Behaghel 1923-1932, IV: 6). 


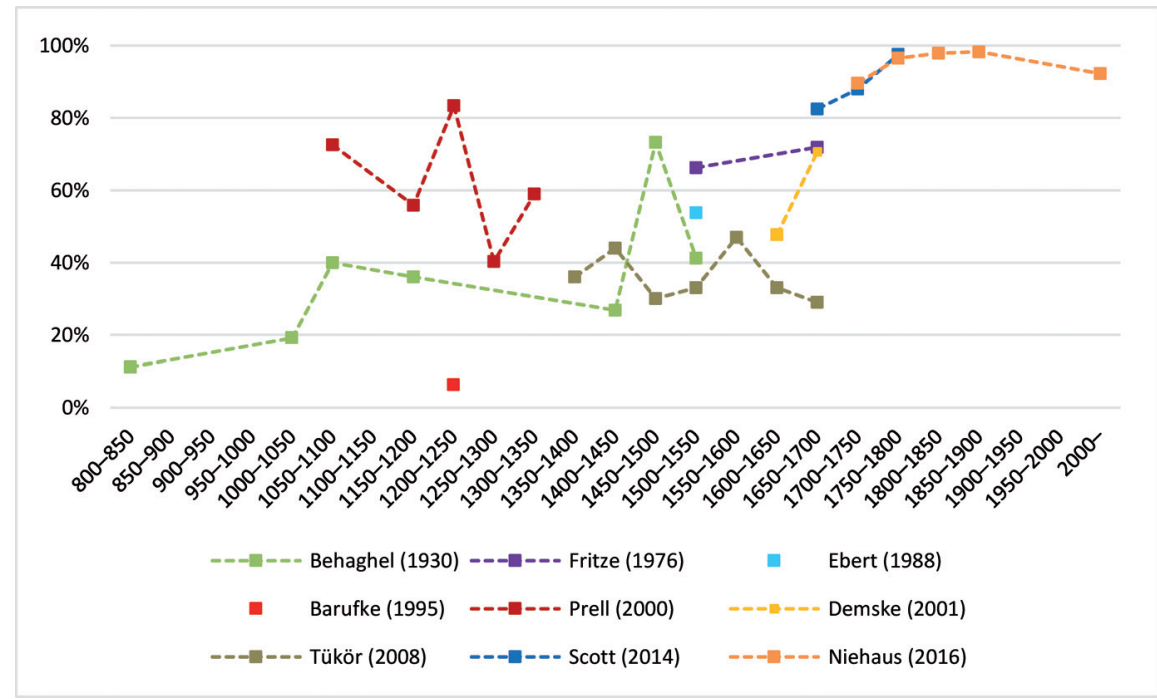

Abb. 1: Überblick über Ergebnisse mehrerer Untersuchungen zur Stellung des adnominalen Genitivs. Abgebildet ist der Anteil der Nachstellung unter allen adnominalen Genitiven

Im Idealfall könnte eine solche Streuung hinsichtlich Raum und Textsorte ein stichprobenartiges Abbild der Variation über die Zeit hinweg liefern, wenn solche Variationsparameter systematisch berücksichtigt werden; ein Problem besteht jedoch darin, dass Zahlen aus unterschiedlichen variationslinguistischen Bereichen oft unreflektiert miteinander verglichen und diachrone Entwicklungen daraus abgelesen werden. So weist etwa Demske (2001) nicht auf die unterschiedliche Herkunft ihrer Zeitungen hin und vergleicht sie mit Eberts (1988) Zahlen, ohne die dort untersuchte Textsorte zu nennen. Dieses Problem pflanzt sich in der Rezeption fort: Nübling et al. (2017: 130-131) greifen die Zahlen aus Ebert (1988) (unter Angabe des Ortes Nürnberg, aber nicht der Textsorte) und Demske (2001) (unter Angabe der Textsorte Zeitung, aber nicht der geographischen Herkunft) auf und zeichnen damit das Bild einer geradlinigen zeitlichen Entwicklung, in der sich die Situation „im 17. Jh. rasant ändert“, obwohl hier unterschiedliche Textsorten aus unterschiedlichen Regionen verglichen werden. Ähnlich wird auf der Grundlage der Zahlen von Fritze (1976) ein Anstieg der Nachstellung von 53\% (um 1500) auf $64 \%$ (um 1700) suggeriert, ${ }^{2}$ obwohl die beiden Teilkorpora sich aus

2 Fritzes Prozentangaben beziehen sich auf voran- und nachgestellte Genitive sowie andere, nicht-genitivische Konstruktionen. Bereinigt um nicht-genitivische Formen liegt die relative Häufigkeit der Nachstellung bei $66 \%$ bzw. 72\%, ist also höher als bei Nübling et al. (2017: 130) angegeben. 
unterschiedlichen Textsorten zusammensetzen, die Unterschiede in der Nachstellung aufweisen und deshalb nicht unmittelbar vergleichbar sind. ${ }^{3}$

Insgesamt wird klar, dass der diachrone Vergleich der verschiedenen Studien und damit eine Gesamtdarstellung der zeitlichen Entwicklung nur mit starken Einschränkungen möglich ist. ${ }^{4}$ Während die in Abb. 1 dargestellten Zahlen eine graduelle, sehr langsame Zunahme der Nachstellung nahezulegen scheinen, wird deutlich, dass ihre relative Häufigkeit zu unterschiedlichen Zeiten deutlichen variationslinguistischen Schwankungen unterlag, abhängig von unterschiedlichen Faktoren. Zudem gibt es einzelne Studien, deren Ergebnisse nicht dem generellen Trend zur zunehmenden Nachstellung entsprechen, so etwa Solling (2011), der in Predigten einen Rückgang postnominaler Formen vom 16. zum 17. Jahrhundert verzeichnet.

In diesem Beitrag wird der Versuch unternommen, zu einer diachron möglichst gleichmäßigen Darstellung der Entwicklung der Stellung des adnominalen Genitivs über annähernd die gesamte Sprachgeschichte des Deutschen hinweg zu gelangen, indem auf eine homogene Basis in Bezug auf Methode, Textsorte und Region gesetzt wird. Ein solcher zeitlicher Längsschnitt ist zwar synchron nicht repräsentativ für den Gebrauch in anderen Textsorten der jeweiligen Zeit, erlaubt jedoch einen klareren, ungetrübten Blick auf zeitliche Entwicklungen, der nicht durch heterogene Untersuchungsmethoden und Korpuszusammenstellungen verzerrt wird. Insbesondere soll hier die Relevanz von internen und externen Variationsfaktoren untersucht werden.

Ein zeitlicher Längsschnitt erfordert ein Korpus, das sich über einen langen Zeitraum erstreckt und dabei hinsichtlich Region, Textsorte und anderer Variationsfaktoren konsistent ist. Als kontinuierlich über die gesamte Sprachgeschichte des Deutschen hinweg überlieferte Textsorten, die diese Eigenschaften aufweisen, kommen praktisch nur zwei Kandidaten infrage: Predigten und Bibelübersetzungen. Während Bibelübersetzungen den Vorteil haben, dass sie durch eine gleichbleibende Textgrundlage ideal für den Vergleich paralleler Textstellen sind, haben sie zum einen den Nachteil, dass sie als Übersetzungen Interferenzen aus der Vorlage und intertextuellen Bezügen aufweisen können; zum anderen

3 So weisen die Herausgeber des Bandes darauf hin, ,daß ein direkter Vergleich der zwei zusammengestellten Gattungskorpora generell nicht vorgenommen werden kann“ (Kettmann \& Schildt 1976: 35).

4 Zusätzlich verschärft wird das Problem der mangelnden Vergleichbarkeit durch die unterschiedlichen Analysemethoden der einzelnen Studien. So zählt etwa Fritze (1976) „nur solche substantivischen attributiven Wortgruppenrelationen [...], die in einen einfachen Aussagesatz mit dem Verb /haben/ transformierbar sind“, was eine große Zahl an Fällen ausschließt. Ebert (1988: 34) wiederum schließt z.B. diskontinuierliche Genitivkonstruktionen aus. 
weisen sie häufig eine besonders markierte Stilistik auf, die sie deutlich von anderen Überlieferungsformen abhebt. Predigten hingegen sind (meist) originär volkssprachliche Texte, deren Prosa als selbständig gelten kann und dabei einen besonderen Bezug zum mündlichen Sprachgebrauch aufweist, insofern sie eine (tatsächliche oder imaginierte) mündliche Kommunikationssituation reflektieren. Dabei unterliegen sie thematischen Schwankungen und inhaltlich-stilistischen zeitlichen Schwerpunkten (man denke nur an die Hochphase der Leichenpredigten um das 17. Jahrhundert). Für die vorliegende Untersuchung wurden Predigten gewählt, da sie neben den genannten Vorteilen eine breitere Überlieferung aufweisen und so eine bessere zeitliche Abdeckung und Auflösung erlauben als Bibelübersetzungen. Dabei sei nochmals darauf hingewiesen, dass eine einzelne Textsorte keinen Anspruch auf Repräsentativität für den übrigen Sprachgebrauch erheben kann.

\section{Das Predigten-Korpus: Daten und Auswertung}

Das für diese Untersuchung verwendete Korpus besteht ausschließlich aus Predigten aus dem oberdeutschen Raum, da nur für diesen Raum eine zeitlich kontinuierliche und relativ gleichmäßige Abdeckung erreicht werden konnte. ${ }^{5}$ Die Quellen aus dem Alt-, Mittel- und frühen Frühneuhochdeutschen (bis 1400) stimmen im Wesentlichen (ein Text für 1300-1350 kam hinzu) mit den in Pickl (2017) verwendeten überein (s. dort für eine genauere Übersicht über die Texte und deren Verteilung). Diese Basis wurde für die Zeit von 1400 bis 1900 mit weiteren Texten ergänzt, sodass insgesamt - mit Ausnahme der Überlieferungslücke vom 9. bis zum 11. Jahrhundert - eine kontinuierliche und ab 1500 (zwischen West- und Ostoberdeutsch) gleichmäßige Abdeckung erreicht werden konnte.

Vorbild für die Struktur des Korpus ist das German Manchester Corpus (GerManC) (vgl. Durrell et al. 2012), dessen oberdeutsche Predigten (1650-1800) auch in das hier untersuchte Korpus eingeflossen sind. Wie bei GerManC wurden auch im Predigten-Korpus Zeitabschnitte von 50 Jahren angesetzt und pro Zeitabschnitt und Region (hier: Ost- und Westoberdeutsch) drei Texte gewählt, von denen je ca. 2.000 Wörter erfasst wurden. Dieses Ziel wurde für Drucke (ab 1500) vollständig erreicht, für Handschriften (bis 1500) jedoch nicht in allen Fällen; dieses Manko ließ sich durch die Heranziehung von zusätzlichen Handschriften

5 Das hier verwendete Korpus ist Teil eines größeren Predigten-Korpus, das sich im Aufbau befindet und ab 1500 auch systematisch mittel- und norddeutsche Quellen berücksichtigt. 
in den meisten Zeitabschnitten ausgleichen. Die Gesamtgröße des Korpus beläuft sich auf 197.802 Wörter (Tab. 1). Die Quellenlage bringt es mit sich, dass über das Althochdeutsche nur sehr eingeschränkt Aussagen getroffen werden können; die wenigen Texte aus dem 9. Jahrhundert (Exhortatio ad plebem christianam und zwei Predigten der Monseer Fragmente) können aufgrund ihrer Nähe zu lateinischen Vorlagen nur sehr bedingt Auskunft über die Stellung des Genitivs geben.

Tab. 1: Übersicht über die Texte des Korpus und ihre zeitliche und räumliche Verteilung

\begin{tabular}{|c|c|c|c|c|c|c|c|c|c|c|c|c|c|c|c|c|c|c|c|c|}
\hline \multirow[b]{3}{*}{ } & \multicolumn{11}{|c|}{ Handschriften } & \multicolumn{8}{|c|}{ Drucke } & \multirow[b]{3}{*}{$\Sigma$} \\
\hline & \multirow{2}{*}{$\begin{array}{l}\dot{\bar{S}} \\
\dot{\sigma} \\
\dot{I} \\
\dot{\leftarrow}\end{array}$} & \multirow[b]{2}{*}{$\sim$} & \multirow{2}{*}{$\begin{array}{l}\dot{I} \\
\dot{I} \\
\dot{I} \\
\dot{N}\end{array}$} & \multicolumn{2}{|c|}{ 12. Jh. } & \multicolumn{2}{|c|}{ 13. Jh. } & \multicolumn{2}{|c|}{ 14. Jh. } & \multicolumn{2}{|c|}{ 15. Jh. } & \multicolumn{2}{|c|}{ 16. Jh. } & \multicolumn{2}{|c|}{ 17. Jh. } & \multicolumn{2}{|c|}{ 18. Jh. } & \multicolumn{2}{|c|}{ 19. Jh. } & \\
\hline & & & & $\stackrel{\dot{x}}{\dot{r}}$ & $\begin{array}{l}\dot{I} \\
\dot{\mathbf{N}}\end{array}$ & $\stackrel{\dot{I}}{\check{r}}$ & $\begin{array}{l}\dot{I} \\
\dot{\mathbf{N}}\end{array}$ & $\stackrel{\dot{x}}{\dot{r}}$ & $\begin{array}{l}\dot{I} \\
\dot{N}\end{array}$ & $\dot{I}$ & $\begin{array}{l}\dot{I} \\
\dot{N}\end{array}$ & $\stackrel{\dot{I}}{\dot{r}}$ & $\begin{array}{l}\dot{I} \\
\dot{N}\end{array}$ & $\stackrel{\dot{I}}{\check{r}}$ & $\begin{array}{l}\dot{I} \\
\dot{N}\end{array}$ & $\stackrel{\dot{I}}{\dot{ }}$ & $\begin{array}{l}\dot{I} \\
\dot{N}\end{array}$ & $\dot{\dot{x}}$ & $\begin{array}{l}\dot{I} \\
\dot{i}\end{array}$ & \\
\hline Wod. & & & & & 5 & 2 & 4 & 4 & 3 & 1 & 3 & 3 & 3 & 3 & 3 & 3 & 3 & 3 & 3 & 46 \\
\hline Ood. & 3 & & 3 & 4 & 6 & 5 & 5 & 2 & 3 & 4 & 2 & 3 & 3 & 3 & 3 & 3 & 3 & 3 & 3 & 61 \\
\hline$\Sigma$ & 3 & & 3 & 4 & 11 & 7 & 9 & 6 & 6 & 5 & 5 & 6 & 6 & 6 & 6 & 6 & 6 & 6 & 6 & 107 \\
\hline 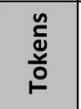 & $\begin{array}{l}\stackrel{\infty}{0} \\
\text { in }\end{array}$ & & $\begin{array}{l}\underset{\text { U్ }}{0} \\
\text { N }\end{array}$ & 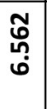 & $\begin{array}{l}\text { ₹̛̣ } \\
\text { ơ }\end{array}$ & 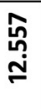 & 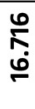 & 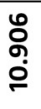 & 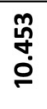 & $\delta_{\infty}^{\delta}$ & $\overbrace{\infty}^{m}$ & 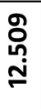 & $\begin{array}{l}\text { مू } \\
\text { న్ } \\
\text { N }\end{array}$ & 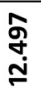 & 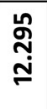 & 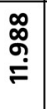 & $\begin{array}{l}\underset{\sim}{\sim} \\
\stackrel{N}{N}\end{array}$ & 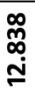 & $\frac{\infty}{\stackrel{\infty}{\underline{m}}}$ & 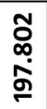 \\
\hline
\end{tabular}

Als adnominale Genitive wurden alle eindeutigen nicht-partitiven Fälle gezählt, in denen der Genitiv aus einer vollständigen Nominalphrase besteht (d.h. determiniert ist oder aus einem Eigennamen besteht). Dies dient der Abgrenzung von Komposita und ambigen Fällen. Ausgeschlossen wurden etwa Fälle, in denen der (formale) Genitiv nur aus einem Substantiv besteht (z.B. Menschen in Menschen sun oder engels in daz engels gesange). Fälle mit vorangestellten artikellosen Eigennamen (hierzu zähle ich auch den Gebrauch von Gottes ohne Artikel; vgl. auch Kopf 2018: 76, 78; eingereicht), wurden hingegen als Genitive gezählt, auch wenn ein mit dem Matrixnomen kongruierender Artikel vorhanden ist, solange sie getrennt geschrieben wurden (z.B. daz gotes lamp); dies war etwa bis 1500 der Fall. Diese Vorgehensweise stellt im Wesentlichen eine Heuristik dar, die sicherstellen soll, dass nur eindeutige Fälle von Genitivattributen gezählt werden (für eine eingehendere Diskussion möglicher Kriterien zur Abgrenzung zwischen Kompositum und Genitivkonstruktion s. Kopf 2018: 110-170). 


\section{Ergebnisse}

Die oben skizzierte Methode führte im Korpus zu einer Gesamtzahl von 3.755 Treffern, die zeitlich ungleich verteilt sind (Abb. $2 ;^{6}$ vgl. Pickl i. E. a).

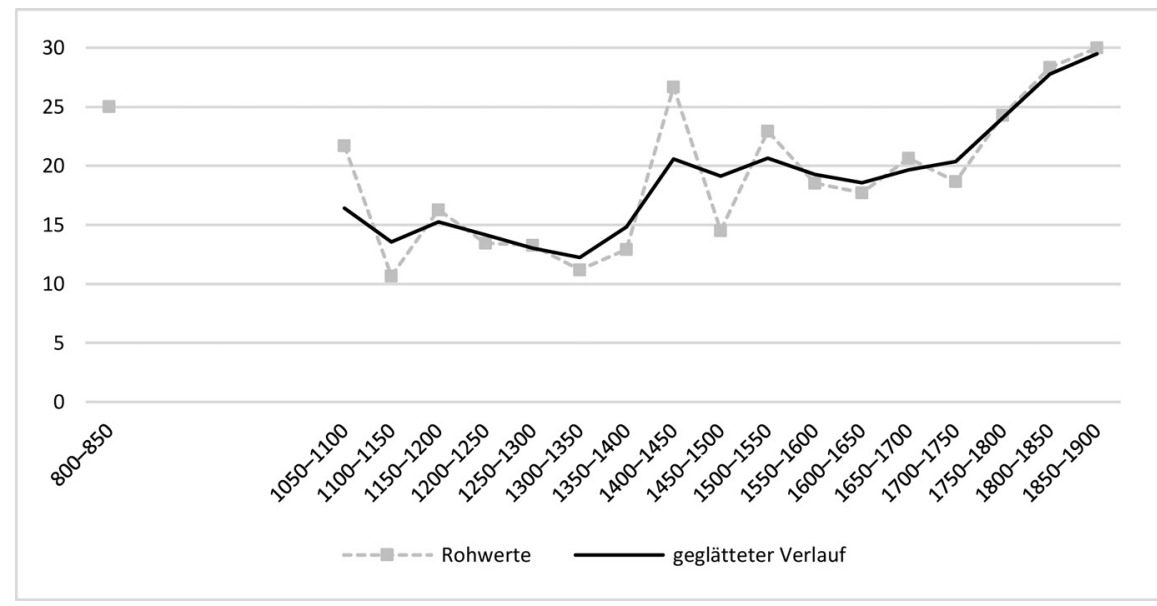

Abb. 2: Verlauf der Frequenz der Genitivattribute pro 1.000 Tokens $(N=3755)$

Auffallend sind hier insbesondere der sprunghafte Anstieg der Verwendung von Genitivattributen um 1400 und der daran anknüpfende graduelle weitere Anstieg im 18. und 19. Jahrhundert, die in Verbindung mit dem Ausbau des Genitivs als Schriftlichkeitsmarker und Standardmerkmal gebracht werden können (vgl. Pickl i. E. a).

Insgesamt sind 787 (21\%) der Genitivattribute vorangestellt und 2.968 (79\%) nachgestellt. Der diachrone Verlauf der Nachstellung wird in Abb. 3 prozentual dargestellt. ${ }^{7}$ Hier fällt zunächst auf, dass die Nachstellung spätestens im Mittelhochdeutschen generell leicht präferiert wird; sie bewegt sich in dieser Zeit etwa

6 Da die Werte zu einzelnen Zeitpunkten statistischen Fluktuationen unterliegen, wurde versucht, dies rechnerisch zu kompensieren und die Einzelwerte zu stabilisieren. Deshalb wird neben den Rohwerten (graue Quadrate, gestrichelte Linie) auch ein leicht geglätteter Verlauf (durchgezogene schwarze Linie) angegeben, der durch temporale Dichteschätzung errechnet wurde und als Trendlinie zu verstehen ist, die von den Einzelwerten abstrahiert und eine realistischere zugrundeliegende Wahrscheinlichkeitsverteilung abbildet. Zur temporalen Dichteschätzung vgl. Blaxter (2017: 106-107) und Pickl (i. E. a, b); für geolinguistische Anwendungen der Dichteschätzung s. Pickl et al. (2014) und Pröll et al. (2015: 175-180).

7 Der Wert des Datenpunkts im allerersten Zeitabschnitt liegt bei 29\%; hier ist jedoch anzumerken, dass es sich um Übersetzungen von lateinischen Vorlagen handelt und deshalb Interferenzen 
zwischen $50 \%$ und $65 \%$. Weiterhin ist auffallend, dass die Entwicklung der Nachstellung nicht graduell verläuft, sondern zwei Schübe - um 1400 und um 1700 - aufweist, und auch einen zwischenzeitlichen Rückgang von einem Hoch (ca. 1400-1550) auf ein Tief (ca. 1550-1700). Dieser Rückgang passt tendenziell zu dem anormal erscheinenden Rückgang der Nachstellung vom 16. auf das 17. Jahrhundert, den Solling (2011) in Predigten feststellt.

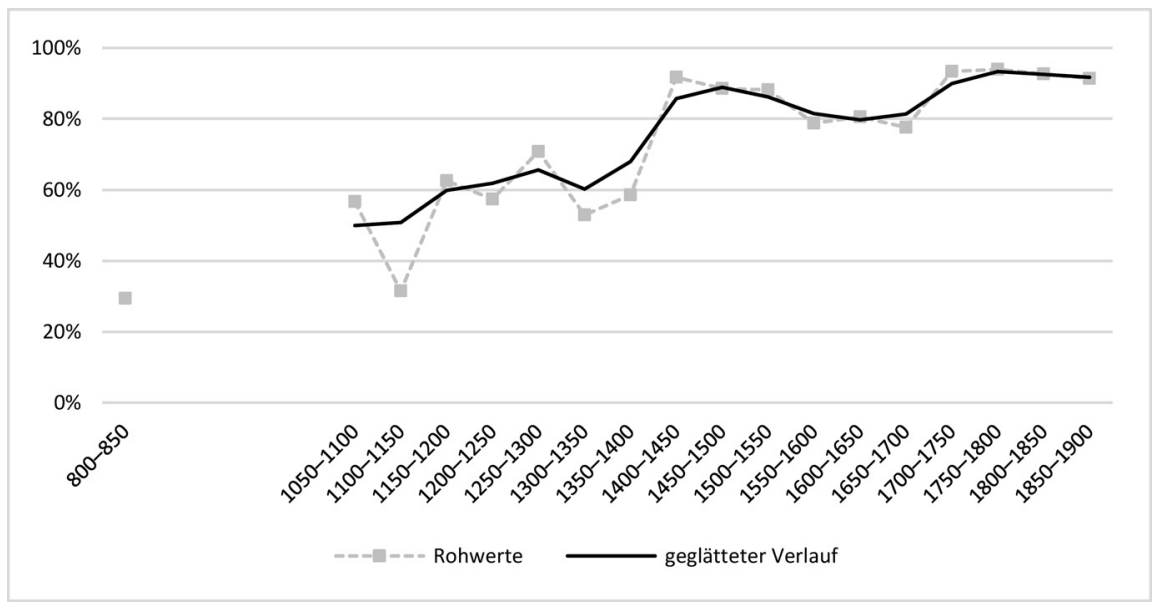

Abb. 3: Zeitlicher Verlauf des Anteils der Nachstellung von Genitivattributen im Predigten-Korpus $(N=3755)$

Im Vergleich mit Abb. 2 ist anzumerken, dass beide Schübe hin zu mehr Nachstellung jeweils mit einem deutlichen Anstieg der Verwendung von Genitivattributen insgesamt verbunden sind. In anderen Worten gehen beide Anstiege in der Genitivverwendung in erster Linie auf einen Anstieg bei den nachgestellten Genitiven zurück (vorangestellte gehen gleichzeitig leicht zurück; vgl. Pickl i. E. a).

Als nächstes soll diese Entwicklung auf den Einfluss von internen Faktoren hin beleuchtet werden, um in der Folge die Relevanz von externen Faktoren zu überprüfen.

nicht auszuschließen sind. Diese Einschränkung gilt für diesen Zeitabschnitt auch in allen weiteren Grafiken. 


\subsection{Interne Faktoren}

Als erster interner Faktor wird zunächst die Belebtheit der Genitiv-Nominalphrase untersucht, deren Relevanz für die Genitivstellung in verschiedenen Formen immer wieder gezeigt wurde (z.B. Ebert 1988; Demske 2001). Gemäß der Belebtheitshierarchie von Silverstein (1976) kommt im nominalen Bereich Eigennamen die höchste Belebtheit zu, gefolgt zuerst von menschlichen und dann nichtmenschlichen Substantiven, und unbelebten Substantiven die geringste Belebtheit. Zunächst werden Genitive von belebten und unbelebten Appellativa miteinander verglichen (Abb. 4). ${ }^{8}$

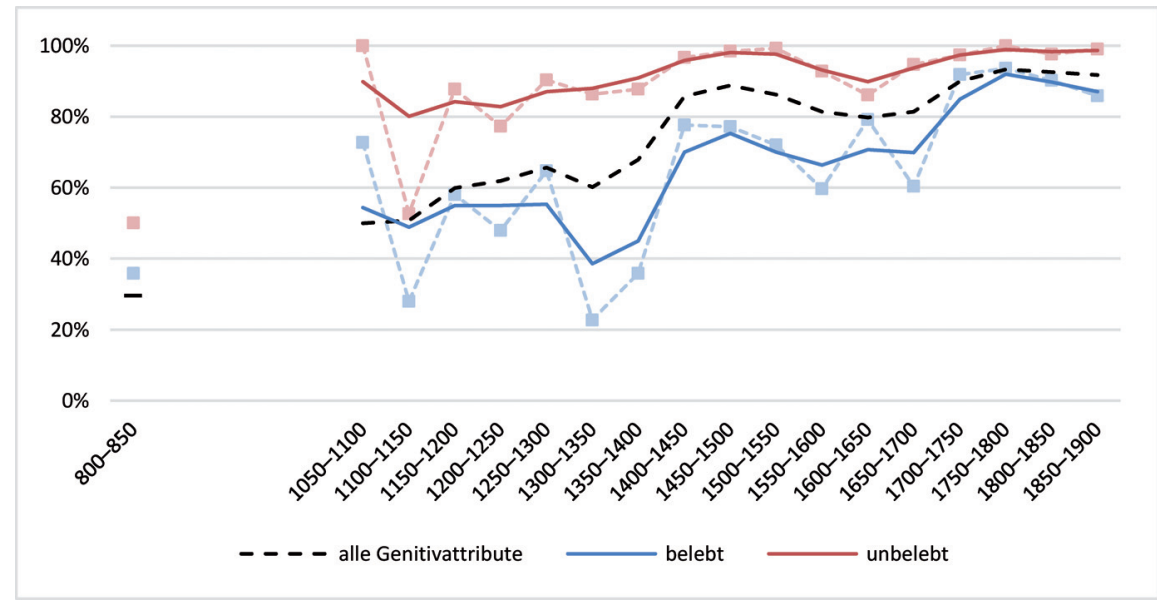

Abb. 4: Anteil der Nachstellung von belebten $(N=917)$ und unbelebten Genitiven $(N=1835)$ (ohne Eigennamen) im Vergleich zu allen Genitivattributen (vgl. Abb. 3)

Belebte und unbelebte Genitive zeigen zu allen Zeiten einen deutlichen Unterschied in der Frequenz der Nachstellung. Diese wird bei unbelebten Genitiven stets deutlich stärker präferiert als bei belebten Genitiven. Unbelebte Genitive stehen seit Beginn des Mittelhochdeutschen zu über $80 \%$ nach, während belebte in derselben Zeit zu etwa gleichen Anteilen vor- und nachstehen und erst ab 1400 eine deutliche Präferenz zur Nachstellung (zunächst um 70\%, später, ab 1700, um $90 \%$ ) entwickeln. In Anbetracht dieser Zahlen ist fraglich, ob erst „,[m]it dem Beginn des 16. Jh.s [...] das Auftreten eines unbelebten Individualnomens präno-

8 Bei den belebten Genitiven handelt es sich im Predigten-Korpus so gut wie ausschließlich um Personenbezeichnungen. 
minal markiert“ ist (Demske 2001: 216 mit Bezug auf Carr 1933). Insgesamt passen die relativ stabilen Zahlen aus dem 16. und 17. Jahrhundert ungefähr zu der von Kopf (2018: 97) aus dem Mainzer Frühneuhochdeutschkorpus berichteten Stagnation mit Schwankungen; allerdings kann ihre Einschätzung, dass „der Stellungswandel [...] weitgehend abgeschlossen [scheint]“, dahingehend ergänzt werden, dass die Nachstellung in Predigten des 18. Jahrhunderts bei Belebtheit nochmals einen deutlichen Schub erfährt.

Eigennamen sind nach Silverstein (1976) hinsichtlich ihrer Belebtheit nicht weiter unterteilt. In der Literatur zur Genitivstellung wird jedoch häufig auf einen Unterschied in Bezug auf die Genitivstellung in Abhängigkeit von der Art des im Genitiv stehenden Eigennamens hingewiesen. So würden Eigennamen, deren Genitiv nach deutschem Flexionsmuster gebildet wird (z.B. die Weisheit Salomons) (= ,deutsche‘ Eigennamen), bevorzugt vorangestellt, während Eigennamen mit lateinischen Genitivendungen (z.B. den Namen Catharinæ) oder flexionslose hebräische Genitive (z.B. ain sun Dauid) (=,fremde‘ Eigennamen) meist nachgestellt aufträten, v.a. vor 1600 (vgl. Reichmann \& Wegera 1993: 337). Das ebenfalls als Eigenname gewertete Gott wird in der Darstellung der Ergebnisse (Abb. 5) gesondert betrachtet.

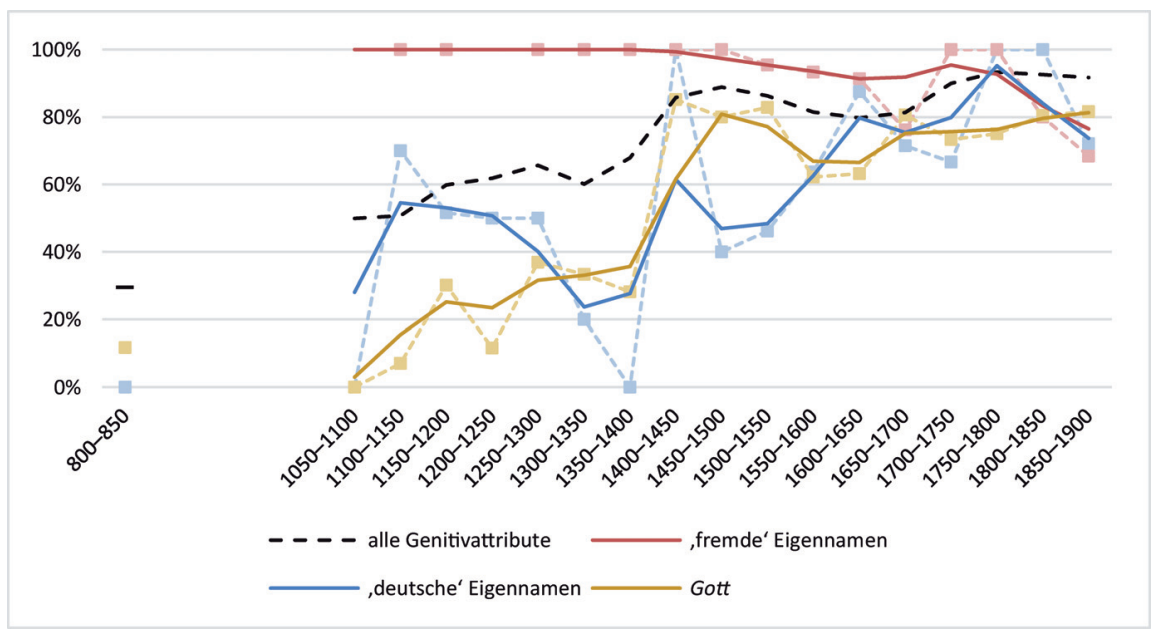

Abb. 5: Anteil der Nachstellung von Genitiven von deutschen $(N=111)$ und fremden Eigennamen $(N=219)$ sowie von Gott $(N=465)$ im Vergleich zu allen Genitivattributen

Die Zahlen aus dem Predigten-Korpus bestätigen einen deutlichen Unterschied in der Genitivstellung bei deutschen und fremden Eigennamen. Letztere sind bis 1500 ausschließlich nachgestellt belegt, die Zahlen der Voranstellung halten sich 
auch in der darauffolgenden Zeit stark in Grenzen. Deutsche Eigennamen kommen insgesamt seltener vor, ihre Rohwerte schwanken deshalb entsprechend; sie sind während des Mittelhochdeutschen bis zu über $50 \%$ nachgestellt und gehen nach einem vorübergehenden Rückgang der Nachstellung ab etwa $1600 \mathrm{zu}$ Werten von über $75 \%$ über. Der Genitiv von Gott zeigt anfangs die stärkste Tendenz zur Voranstellung und geht nur allmählich zur Nachstellung über; ab 1400 ist er zu ca. $80 \%$ nachgestellt und bleibt nach einem vorübergehenden Rückgang um 1600 bei ähnlichen Werten. ${ }^{9}$ Bemerkenswert ist hier vor allem, dass die häufig konstatierte Kontinuität der Voranstellung der Eigennamen vom Althochdeutschen bis heute mit diesen Zahlen nicht zu bestätigen ist - im Gegenteil stehen diese im vorliegenden Korpus im Mittelhochdeutschen häufig nach und etwa ab dem 16. Jahrhundert sogar überwiegend. Aufgrund der geringen Fallzahlen ist jedoch Vorsicht beim Ziehen von Schlussfolgerungen geboten, wenngleich die weitgehend parallele Entwicklung in Richtung Nachstellung bei deutschen Eigennamen und Gott auf eine gewisse Belastbarkeit der generellen Tendenz hindeutet.

Weitere potentielle interne Faktoren betreffen die syntaktische Struktur der Genitivkonstruktion. Hier geht es vor allem um die Komplexität der Genitivphrase (vgl. Kopf 2018: 99-100), die etwa in der Attribuierung durch Adjektive oder untergeordnete Genitivattribute bestehen kann. Die häufigste Form ist im vorliegenden Korpus mit 56\% die Kombination aus Determinator und Substantiv. Auch die Struktur der durch den Genitiv modifizierten Nominalphrase ist relevant (vgl. z.B. Bassola 1985). Nicht durch Adjektive modifizierte Matrixnomina stellen hier mit $83 \%$ klar den Normalfall dar. Bei der vorliegenden Untersuchung (Abb. 6) werden die von diesen Normalfällen (z.B. der geist des herrn oder der welt erlösung) abweichenden Fälle betrachtet und danach unterschieden,

(1) ob das Matrixnomen durch ein Adjektiv modifiziert ist (z.B. den richtigen Fußpfad deß Heyls), und seitens des Genitivs,

(2) ob er ,nackt' ist, d.h. nur aus einem Eigennamen (z.B. Luthers gäist) oder einem (modifizierten) Substantiv ohne Artikel(-wort) (z.B. der warheit Gotlicher fchrifft) besteht,

(3) ob er durch ein Adjektiv modifiziert ist (z.B. die ordnung der heyligen kirchen), und

9 Reichmann \& Wegera (1993: 337) vermuten hinter einer frühneuhochdeutschen Tendenz zur Nachstellung von Nomina sacra lateinischen Einfluss; Kopf (2018: 102) sieht zusätzlich eine „grammatische Differenzierungsstrategie“, „die bewirkt, dass die Gottesbezeichnung nicht mit den Menschenbezeichnungen zusammenfällt“. In Anbetracht der Vergleichswerte zwischen Gott und übrigen Eigennamen sowie belebten Substantiven scheinen die Zahlen aus dem PredigtenKorpus solche Zusammenhänge nicht nahezulegen. 
(4) ob er komplex ist, d.h. aus mehr als einem Substantiv besteht, etwa bei Koordination (z.B. die vermählung Marie vnd Joseph) oder Modifikation des Genitivs durch einen davon abhängigen Genitiv (z.B. die Erfullung der trawrigen Weissagung Christi). ${ }^{10}$

,Nackte‘ Genitive zeigen hier als einziger Typ eine Tendenz zur Voranstellung (verglichen mit der Gesamtentwicklung). Der Verlauf ähnelt stark dem von Gott (Abb. 5), was nicht überrascht, da über die Hälfte (55\%) der ,nackten“ Genitive auf Gott zurückgeht. Alle übrigen hier beobachteten Faktoren bewirken verstärkte Nachstellung des Genitivs, vor allem Matrixnomina mit Adjektiv. Diese letztere Konstellation führt bis etwa 1500 sogar zur fast ausschließlichen Nachstellung, was Behaghels „Gesetz der wachsenden Glieder“ (vgl. Anm. 1) zuwiderzulaufen scheint.

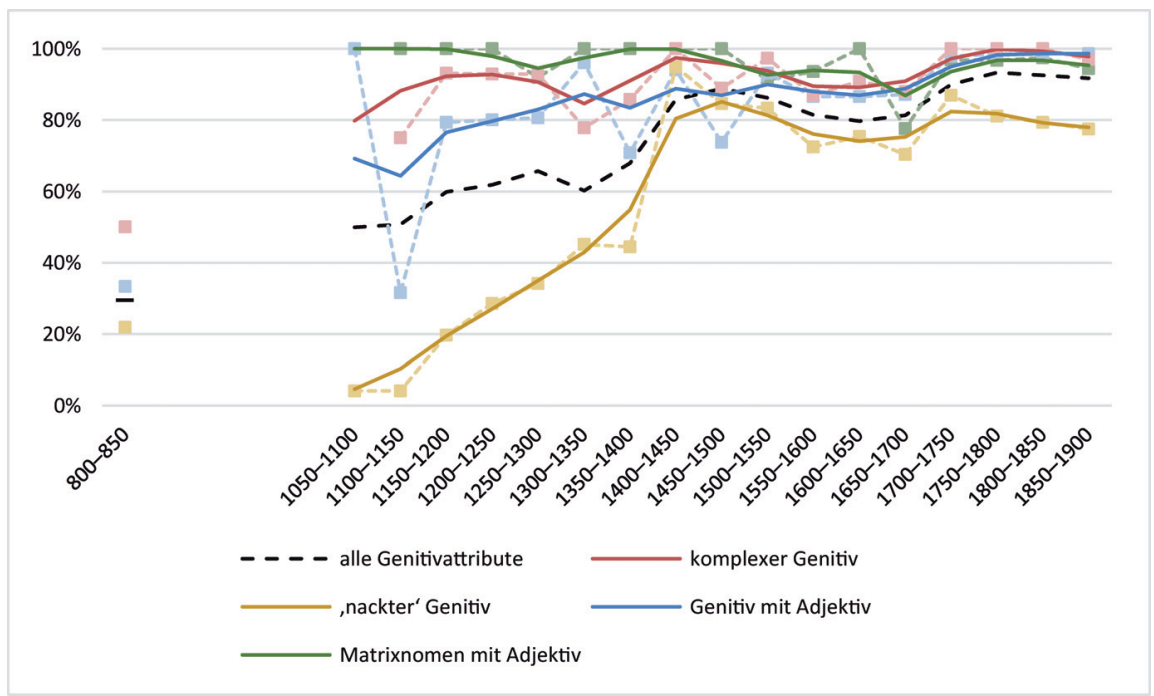

Abb. 6: Anteil der Nachstellung von ,nackten' Genitiven $(N=839)$, komplexen Genitiven $(N=374)$, Genitiven mit Adjektiv(en) $(N=813)$ und bei Matrixnomen mit Adjektiv(en) $(N=655)$ im Vergleich mit allen Genitivattributen

10 Diese Kategorien sind nicht exhaustiv (die beiden genannten Normalfälle sind nicht abgedeckt) und schließen einander nicht prinzipiell gegenseitig aus; so kann ein konkreter Fall z.B. unter Kategorie (1) und Kategorie (3) gezählt werden. 
Über alle bisher betrachteten internen Faktoren hinweg zeigt sich eine interessante Struktur, die praktisch alle Genitivtypen betrifft und zwischen 1500 und 1700 zu einem vorübergehenden Rückgang der Nachstellung führt. Es ist plausibel, als Ursache einen weiteren Faktor zu vermuten, der bis hierher nicht in Betracht gezogen wurde. Hierfür kommt zum Beispiel die zeitlich ungleiche Verteilung von semantischen Kategorien in Betracht. Zeigen bestimmte semantische Kategorien, die zur Voranstellung neigen, ein zeitlich gehäuftes Auftreten, könnte dies den beobachteten Effekt erklären: „Treten in einem Zeitschnitt z.B. besonders viele Titel auf, so ist zu vermuten, dass die Nachstellung insgesamt einen geringeren Anteil ausmacht als dies sonst der Fall wäre.“ (Kopf 2018: 95) Die Häufigkeit von Titeln (die aufgrund der Häufigkeit von Leichenpredigten im fraglichen Zeitraum ein plausibler Kandidat zu sein scheint) kommt aufgrund ihrer zeitlichen Verteilung im Predigten-Korpus dafür jedenfalls nicht infrage.

Möglicherweise liegt die Erklärung jedoch in einem weiteren internen Faktor, der weder die semantischen Kategorien des Genitivs noch seine syntaktische Struktur betrifft, sondern die semantische Relation zwischen Genitiv und Matrixnomen. Kopf (2018: 104-106) zeigt auf der Grundlage von Zahlen aus Bassola (1985) und Lanouette (1998), dass semantische Relationen im 15. und 16. Jahrhundert eine große Rolle bei der Genitivstellung spielen konnten. Interessant ist hier besonders der Unterschied zwischen Subjekts- (z.B. jenen Ausspruch des Propheten) und Objektsgenitiven (z.B. die Erhaltung seines Lebens), der auch im Predigten-Korpus festzustellen ist (Abb. 7). Ein gewisser Unterschied in der Nachstellungspräferenz beider Typen ist etwa ab 1200 festzustellen. Um 1400 nähern sich die Werte wieder stark an, um dann umso deutlicher auseinanderzugehen: In der fraglichen Zeit zwischen 1500 und 1700 ist es vor allem der Subjektsgenitiv, der im Vergleich mit übrigen Genitiven eine neue, deutliche Tendenz zur Voranstellung ausbildet, die im 17. Jahrhundert stärker ausgeprägt ist als bei allen anderen untersuchten Genitivtypen. Umgekehrt ist der Objektsgenitiv in derselben Zeit fast ausschließlich nachgestellt, sodass er fast keine Anzeichen des sonst allumfassenden Rückgangs im 16. und 17. Jahrhundert zeigt. Die Separation von Subjekts- und Objektsgenitiv im Hinblick auf ihre Stellung kann also nicht durch die anderen Faktoren erklärt werden; ${ }^{11}$ eher steht zu vermuten, dass die semantischen Relationen durch ihre Assoziationen mit anderen Genitivtypen Einfluss auf deren Stellungsentwicklung in dieser Zeit gehabt hat.

11 Kopf (2018: 105-106) vermutet Belebtheit als hinter der Verteilung der Nachstellung bei Subjekts- und Objektsgenitiven stehenden Faktor. Da jedoch Subjekts- und Objektsgenitive in den Predigten im 17. Jahrhundert eine etwas stärkere Separation bei der Voran- und Nachstellung erreichen als belebte und unbelebte Genitivphrasen (Abb. 4), scheint zumindest in diesem Zeitabschnitt eine autochthone Entwicklung bei der Genitivrelation vorzuliegen. 


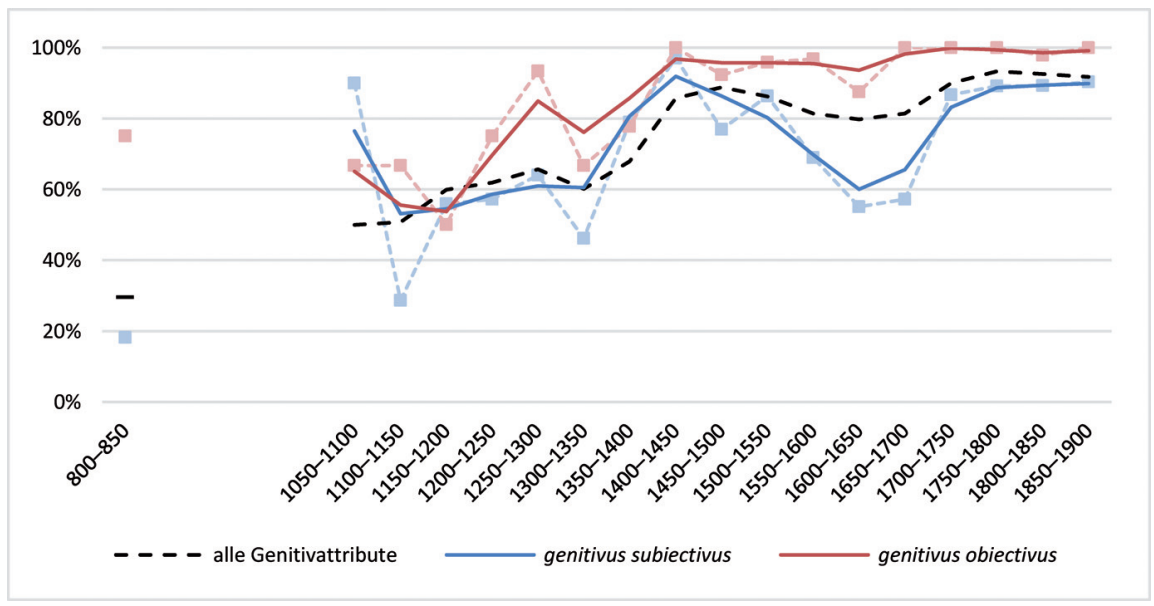

Abb. 7: Anteile der Nachstellung bei Subjekts- $(N=437)$ und Objektsgenitiv $(N=366)$ im Vergleich mit allen Genitivattributen

\subsection{Externe Faktoren}

Neben internen Faktoren kommen auch externe Faktoren in Betracht. Neben der Region bietet sich hier - nicht zuletzt aufgrund der untersuchten Textsorte - der Faktor Konfession an. Soziale Variation und Textsortenvariation können mit diesem Korpus hingegen nicht untersucht werden.

Fritze (1976: 460) identifiziert in ihrem Korpus aus verschiedenen Textsorten (um 1500 und um 1700) regionale Unterschiede in der Genitivstellung. So bevorzugt zu beiden Zeitschnitten das Ostmitteldeutsche die Nachstellung deutlicher (zu 74\% bzw. 78\%) als die anderen Regionen. ${ }^{12}$ Auch innerhalb des Oberdeutschen finden sich Unterschiede in der Frequenz der Nachstellung (mit 62\% bzw. $68 \%$ im Ostoberdeutschen und 68\% bzw. 73\% im Westoberdeutschen). Im Predigten-Korpus finden sich keine deutlichen Unterschiede zwischen dem Ostund dem Westoberdeutschen (Abb. 8), jedenfalls nicht stabil über längere Zeiträume hinweg. So scheint im 13. und frühen 14. Jahrhundert das Westoberdeutsche etwas eher zur Nachstellung zu neigen als das Ostoberdeutsche, während es im 17. und Anfang des 18. Jahrhundert umgekehrt ist.

12 Fritzes Zahlen wurden dahingehend angepasst, dass die Prozentwerte den Anteil an allen Genitivattributen (und nicht an Genitivattributen und alternativen Formen) wiedergeben (vgl. Anm. 2). 


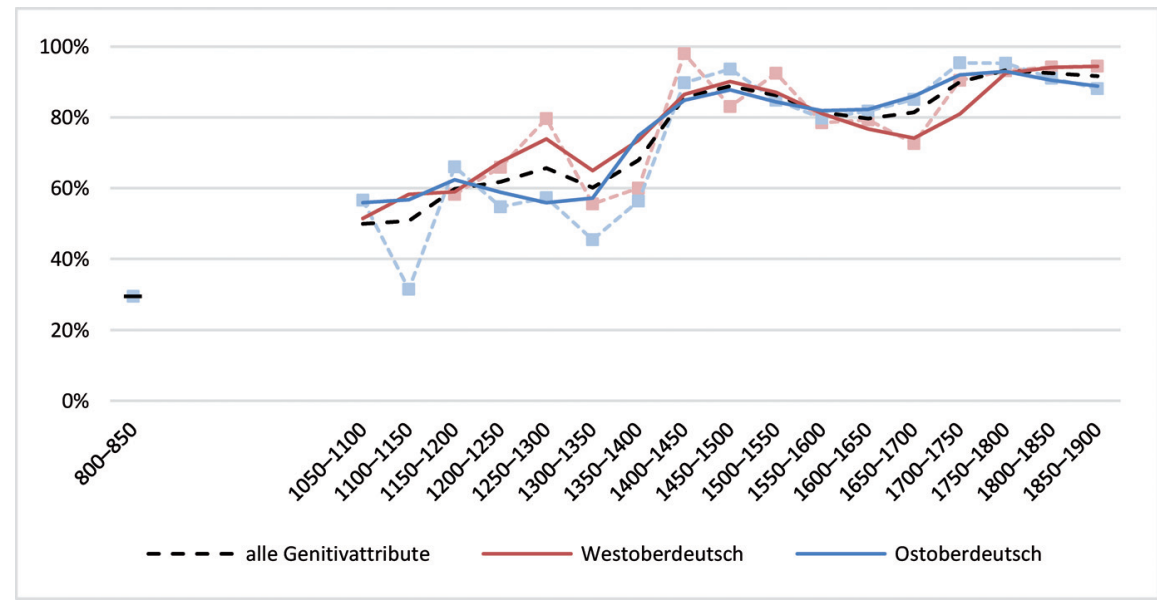

Abb. 8: Anteile der Nachstellung in westoberdeutschen $(N=1740)$ und ostoberdeutschen Predigten $(N=2015)$ im Vergleich mit allen Genitivattributen

Der Faktor der Konfessionalität kann bei Predigten als naheliegende Größe betrachtet werden (s. etwa Habermann 2012 für eine einschlägige Studie; vgl. auch Macha 2014: 120-122). Sie steht jedoch in einem oft schwierig aufzulösenden Verhältnis zur Regionalität (vgl. Macha 2014: 210-211; Wandt 2012). In Abb. 9 sind die Anteile der Nachstellung getrennt nach katholischen und protestantischen Predigten abgebildet (auf die Einzeichnung des Verlaufs für alle Genitivattribute wurde hier verzichtet, da die Linie bis 1500 mit der der katholischen Predigten identisch ist). Interessanterweise besteht in der relevanten Zeit tatsächlich ein durchgehender Unterschied dahingehend, dass katholische Texte geringfügig, aber zeitlich konsistent (mit wenigen Ausnahmen in den Rohwerten) eher zur Nachstellung neigen als katholische Texte. Bemerkenswert ist dies vor allem deshalb, weil dieser Befund auf der Grundlage der bisherigen Ergebnisse nicht durch regionale Unterschiede (Abb. 8) erklärt werden kann. Nicht ausgeschlossen werden kann derzeit die Möglichkeit, dass der Unterschied auf der unterschiedlich ausgeprägten Tendenz zur Verwendung bestimmter semantischer Klassen beruht; dies scheint jedoch zumindest auf den ersten Blick nicht der Fall zu sein (so verwenden etwa protestantische Predigten im relevanten Zeitraum etwas mehr unbelebte Genitive als katholische, was eher einen umgekehrten Effekt erwarten lassen würde). 


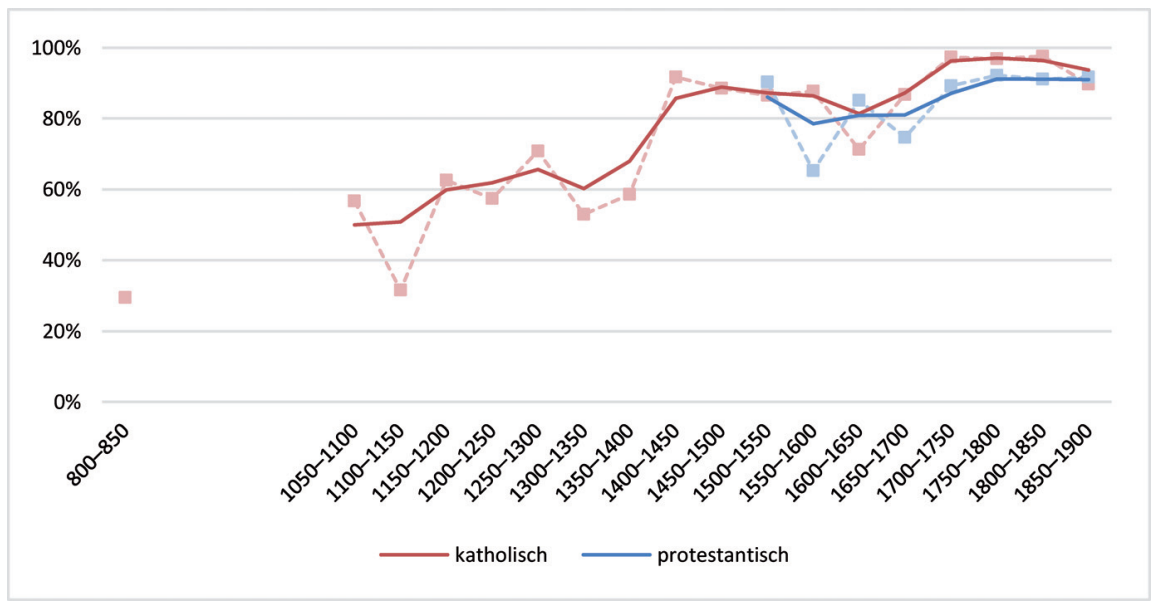

Abb. 9: Anteile der Nachstellung in katholischen $(N=2626)$ und protestantischen Predigten $(N=1129)$

\section{Diskussion}

Die diachronen Befunde führen zunächst vor Augen, dass der Anstieg der relativen Frequenz der Nachstellung mit einer Zunahme der absoluten Frequenz der Verwendung des adnominalen Genitivs insgesamt verbunden ist. Weiterhin ist in Abhängigkeit von (einer Auswahl von) internen und externen Faktoren festzustellen, dass die Entwicklung der Genitivstellung in erster Linie in einem Wandel der relativen Frequenzen der beiden Grundvarianten Voran- und Nachstellung und ihren zugrundeliegenden Wahrscheinlichkeiten besteht. Diese Wahrscheinlichkeiten werden jeweils beeinflusst durch verschiedene Faktoren und entwickeln sich so für verschiedene Typen von Genitivkonstruktionen unterschiedlich. Ein kategorischer Wandel der Genitivstellung kann insgesamt gesehen aus den Ergebnissen nicht abgeleitet werden: Zu keinem Zeitpunkt ist eine der Grundvarianten obligatorisch oder obsolet. Dies gilt nicht für einzelne Typen: So kommen etwa fremde Eigennamen vor 1500 und Genitive, deren Matrixnomen durch ein Adjektiv modifiziert ist, vor 1250 (bzw. vor 1500 fast) ausschließlich nachgestellt vor. Ähnlich sind Objektsgenitive nach 1650 (fast) ausschließlich nachgestellt.

Eine besondere Herausforderung besteht hier darin, etwaige Interaktionen zwischen den einzelnen Faktoren herauszuarbeiten und klar zu beziffern (vgl. auch Kopf 2018: 104, 107). Dies ist aufgrund der Komplexität der beteiligten Faktoren und ihrer Kombinationen hier nur ansatzweise möglich gewesen; dabei wurden die externen Faktoren der Textsorten- und sozialen Variation noch gar 
nicht berücksichtigt. Als Beispiel sei nur die Frage genannt, ob die anfängliche Tendenz der ,nackten' Genitive zur Voranstellung (Abb. 6) als primär anzusehen ist oder die der Eigennamen bzw. des Lexems Gott (Abb. 5), d.h. ob es sich hier um eine syntaktische oder um eine lexikalische Beschränkung handelt (Oubouzar 1997: 235 entscheidet für das Althochdeutsche zugunsten der Syntax). Im Gegensatz dazu scheint der Unterschied zwischen Subjekts- und Objektsgenitiven (Abb. 7) im 17. Jahrhundert relevanter für die Genitivstellung gewesen zu sein als andere Faktoren (vgl. Anm. 11), die in dieselbe Richtung wirken. Multifaktorielle Analysen könnten hier in Zukunft zu differenzierteren Erkenntnissen führen.

Insgesamt scheint die Charakterisierung der Entwicklung als gestaffelter Stellungswandel (vgl. z.B. Behaghel 1930: 49-50; Demske 2001: 265-266; Nübling et al. 2017: 130) zu kurz zu greifen, da die Veränderung der Frequenzen hin zur Nachstellung nicht schrittweise erfolgt und beide Varianten zu allen untersuchten Zeitpunkten unter fast allen untersuchten Bedingungen belegt sind. Die beobachtbaren Veränderungen lassen sich besser als kollektive Verschiebung der Frequenzen der beiden Varianten in Abhängigkeit von verschiedenen Faktoren beschreiben. Diese Frequenzen haben sich teils drastisch geändert, und aufgrund unterschiedlicher Ausgangsfrequenzen kann der Eindruck einer gestaffelten Entwicklung entstehen, wenn man nur wenige Zeitabschnitte betrachtet. Der Wandel besteht somit einerseits in der Veränderung der Grundwahrscheinlichkeit von Voran- bzw. Nachstellung, andererseits in der sich ändernden Wirkstärke und -richtung der jeweiligen Einflussfaktoren und den daraus resultierenden Frequenzen.

Die Entwicklungen lassen sich somit besser als variationslinguistische Verschiebungen beschreiben denn als Stellungswandel oder als „Rechtsversetzung“ (Nübling et al. 2017: 129-130). In chronologischer Hinsicht kann vor allem die damit in Zusammenhang stehende Vorstellung nicht aufrechterhalten werden, dass bis weit ins Mittelhochdeutsche hinein praktisch keine Nachstellung von adnominalen Genitiven vorkam (vgl. etwa Nübling et al. 2017: 129-130), die sich schon aufgrund bisheriger Forschung nicht halten lässt (z.B. Oubouzar 1997). Dies ist im untersuchten Korpus nur bei deutschen Eigennamen der Fall, alle anderen Typen kommen zu nicht geringen Anteilen auch früh in Nachstellung vor. Der Anteil der Nachstellung liegt insgesamt zu Beginn des Mittelhochdeutschen bei etwa $50 \%$ und steigt in der Folge weiter an; lediglich der Genitiv von Gott und andere ,nackte‘ Genitive zeigen im Mittelhochdeutschen überhaupt eine klare Tendenz zur Voranstellung. Die Entwicklung während des Frühneuhochdeutschen und Neuhochdeutschen ist vor allem deshalb interessant, weil sie nicht geradlinig ist. Der Anstieg in der Nachstellung um 1400 ist fast abrupt, und zwischen 1500 und 1700 erfolgt ein vorübergehender leichter Rückgang zugunsten der Voranstellung. Während des Hochs zwischen 1400 und 1450 konvergieren 
fast alle Typen bei ca. 90\% Nachstellung; belebte Substantive und deutsche Eigennamen gehen in dieselbe Richtung, jedoch weniger ausgeprägt. Das darauf folgende Tief scheint hauptsächlich auf das Konto von Subjektsgenitiven zu gehen, die einen neuen Kontrast zu Objektsgenitiven ausbilden. Bemerkenswert ist der Befund, dass Eigennamen und andere Genitive ohne Determinator im Verlauf des Frühneuhochdeutschen - bei aller aufgrund der Fallzahlen gebotenen Vorsicht - eine deutliche und nachhaltige Tendenz zur Nachstellung entwickeln; die gängige Darstellung ist hier, dass diese diachron seit dem Althochdeutschen „in der Prästellung verharren“ (Nübling et al. 2017: 131) bzw. „die ältere Konstruktion bewahren - [...] bis heute“ (Kopf 2018: 95; vgl. auch Demske 2001: 220).

Die Frage, wieso sich langfristig im Deutschen die Nachstellung als Normalfall durchgesetzt hat, wurde häufig versucht zu beantworten; Donhauser (1998) spricht hier vom „Genitivproblem“. Die vorgebrachten Erklärungsansätze sollen hier nicht im Detail wiedergegeben werden; hierfür sei auf die zusammenfassenden Darstellungen in Demske (2001: 220-222) und Kopf (2018: 89-93) verwiesen. Dennoch seien hier kurz einige - meines Wissens neue - Aspekte hinzugefügt.

Spätestens in mittelhochdeutscher Zeit gibt es einige Typen, die (praktisch) ausschließlich auf Nachstellung beschränkt sind (lateinische Eigennamen, Matrixnomen mit Adjektiv, indefinite Matrixnomen ${ }^{13}$ ), und andere, die die Nachstellung deutlich präferieren (komplexe Genitive, Genitive mit Adjektiv); es gibt jedoch keine Typen, die auf Voranstellung beschränkt sind oder diese deutlich präferieren. Die wenigen Fälle, bei denen zu Beginn des Mittelhochdeutschen Voranstellung (fast) ausschließlich vorkam (Gottes und andere ,nackte' Genitive), erwarben wohl durch Analogie schnell auch die Möglichkeit zur Nachstellung. Dies können wir so verstehen, dass Voranstellung unter bestimmten Bedingungen ausgeschlossen war, Nachstellung jedoch nicht - ein deutlicher Vorzug der postnominalen Position. Nachdem Nachstellung auch für die traditionell vorangestellten Typen zur Strukturoption geworden war, setzte ein genereller Drift Richtung Nachstellung ein, der sich in den Entwicklungen während des Mittelhochdeutschen beobachten und dadurch erklären lässt, dass Nachstellung als ,sichere Option“ generell attraktiver und damit zunehmend auch für ursprünglich eher vorangestellte Typen akzeptabel wurde. Hinzu kamen mindestens zwei strukturelle Beschränkungen, die Voranstellung bei bestimmten Genitivtypen ausschloss.

13 Dieser letzte Strukturtyp wurde in diesem Beitrag nicht eigens untersucht, da er - je nach Blickwinkel - Voranstellung ausschließt oder vorangestellte Genitive definite Lesart zur Folge haben. Fälle mit vorangestelltem Genitiv und unbestimmtem Artikel sind zwar belegt (vgl. z.B. Demske 2001: 225-226), kommen im vorliegenden Korpus aber nicht vor. 
Zum einen werden zum Kopfnomen gehörende Determinatoren mit einem vorangestellten Genitiv zunehmend unvereinbar (Pavlov 1983: 61; Kopf 2018: 8082); Konstruktionen mit pränominalem Genitiv werden automatisch als definit gelesen (Reichmann/Wegera 1993: 336). Um Indefinitheit des Kopfnomens auszudrücken, ist also Nachstellung die einzige Option. Zweitens ist die Bildung von Genitivketten durch Kombination von Genitiven in Voranstellung nur stark eingeschränkt möglich; bei Nachstellung ist eine theoretisch beliebig lange Aneinanderreihung von Genitiven möglich. Die zunehmende Verwendung von komplexen Genitivattributen, deren Zahl sich vom Mittelhochdeutschen (mit durchschnittlich fünf Fällen pro 1000 Tokens) zum Frühneuhochdeutschen (mit im Schnitt 15 Fällen pro 1000 Tokens) verdreifachte, war nur mit der vermehrten Verwendung nachgestellter Genitive möglich.

Der nachgestellte Genitiv unterlag also nicht den Beschränkungen wie der vorangestellte und konnte deshalb wesentlich flexibler eingesetzt werden. Er wurde zum ,Allrounder‘, dessen Einsatz praktisch immer möglich war. Dies allein dürfte bereits zu seiner zunehmenden Bevorzugung geführt haben; weitere in der Literatur diskutierte Faktoren mögen ebenfalls dazu beigetragen haben. Etwa um 1400 fand ein qualitativer Umbruch statt: Nachstellung wurde für alle Typen mit meist über 90\% - zum Normalfall. Dadurch wurden die Unterschiede zwischen verschiedenen Typen nivelliert, was den Weg für das Entstehen neuer Regularitäten ebnete. Dies zeigt sich etwa daran, dass ab jetzt Typen, die bislang kategorisch nachgestellt wurden (wie lateinische Namen und Genitive bei Matrixnomen mit Adjektiv), auch vorangestellt werden konnten; umgekehrt wurden Typen, die durchaus auch vorangestellt werden konnten, auf Nachstellung festgelegt (wie Objektsgenitive). Kurz gesagt fand zu dieser Zeit ein Aufbrechen und Umbrechen des Beschränkungssystems statt, das wohl dadurch zu erklären ist, dass alte Variationsmuster aufgrund der um sich greifenden Nachstellung nicht mehr erkannt wurden und dadurch nivelliert wurden, und sich unter anderem bei den Genitivrelationen bemerkbar machte.

Hinzu kommt ein weiterer Umstand, der in bisherigen Darstellungen zur Entwicklung der Genitivstellung wenig Beachtung fand, aber meiner Ansicht nach wesentlich ist. Es handelt sich um die bekannte Tatsache, dass der adnominale Genitiv wohl seit frühneuhochdeutscher Zeit im gesprochenen Deutsch, insbesondere in den Dialekten, weitestgehend geschwunden ist; „nur in der auf der Schriftsprache fußenden Umgangssprache hat sich dieser Gen. erhalten“ (Dal 2014: 25-26). Laut Behaghel (1923-1932, IV: 189) ist „in der lebendigen Rede etwa seit dem Beginn der neueren Zeit der Genitiv dem Untergang verfallen“; er datiert den Verlust des Genitivs im gesprochenen Deutsch auf das 15. Jahrhundert (Behaghel 1923-1932, I: 479-483) und begründet diese Einschätzung unter anderem mit sich häufenden Fällen der „Unsicherheit seines Gebrauchs“ (Behaghel 1923- 
1932, I: 480) in dieser Zeit. Akzeptiert man diese zeitliche Einordnung, die meines Wissens unbestritten geblieben ist, so handelt es sich beim adnominalen Genitiv ab dem 15. Jahrhundert um ein Merkmal der geschriebenen Sprache, das nicht mehr auf oralen Kompetenzen beruht, sondern im Gegenteil sogar zum Schriftlichkeits- und später Standardmarker wurde (vgl. Pickl i. E. a). In diesem Sinne unterscheidet sich die beobachtete Genitivverwendung ab dem 15. Jahrhundert von der früheren dadurch, dass sie nicht mehr im mündlichen Sprachgebrauch verankert ist, sondern eine autochthon schriftsprachliche Entwicklung darstellt. Der Schwund des Genitivs im Gesprochenen ist nicht nur eine Erklärung für die „Unsicherheit seines Gebrauchs“ (Behaghel 1923-1932, I: 480), sondern muss auch als weiterer Grund für das Kollabieren der Variationsmuster im 15. Jahrhundert in Betracht gezogen werden: Als die entsprechende Kompetenz im Gesprochenen verloren ging, wurden die bis dahin wirkenden Beschränkungen nicht mehr erkannt; die Variationsmuster änderten sich zugunsten der vielseitig einsetzbaren Nachstellung.

Zusammengefasst kann die Entwicklung der Genitivstellung im Deutschen als Geschichte ihrer Variationsbeschränkungen beschrieben werden. Verschiedene Faktoren verhinderten die Voranstellung unter bestimmten strukturellen Bedingungen, was in der Folge zu einer typenübergreifenden Tendenz zur Nachstellung führte. Diese kulminierte im 15. Jahrhundert, zu einer Zeit, als der Genitiv in der gesprochenen Sprache verlorenging, sich aber gleichzeitig als schriftsprachliches Merkmal etablierten konnte. In dieser Situation der schwindenden oralen Genitivkompetenz konnten alte Variationsmuster nicht mehr verlässlich reproduziert werden, zumal variationslinguistische Unterschiede zwischen verschiedenen Typen durch die um sich greifende Nachstellung teilweise eingeebnet wurden. Durch den Umbruch hin zur durch bisherige Beschränkungen bereits begünstigen Nachstellung als neuem Normalfall wurde das bestehende Beschränkungssystem aufgelöst. Dies ermöglichte eine neue Dynamik, die sich nur (bzw. primär) in der Schriftsprache abspielte und neue Beschränkungsrelationen ausbildete, die zu neuen Variationsmustern führte. Dabei ermöglicht das hier verwendete Längsschnitt-Korpus einen einzigartigen Blick auf den diachronen Wandel der Genitivstellung in Abhängigkeit von internen und externen Faktoren.

\section{Literatur}

Ágel, Vilmos (2000): Syntax des Neuhochdeutschen bis zur Mitte des 20. Jahrhunderts. In Werner Besch, Anne Betten, Oskar Reichmann \& Stefan Sonderegger (Hrsg.), Sprachgeschichte. Ein Handbuch zur Geschichte der deutschen Sprache und ihrer Erforschung. 2. Teilband, 1855-1903. Berlin, New York: De Gruyter. 
Barufke, Birgit (1995): Attributstrukturen des Mittelhochdeutschen im diachronen Vergleich. Hamburg: Buske.

Bassola, Peter (1985): Wortstellung im Ofner Stadtrecht: Ein Beitrag zur frühneuhochdeutschen Rechtssprache in Ungarn. Berlin: Akademie-Verlag.

Behaghel, Otto (1923-1932): Deutsche Syntax. Eine geschichtliche Darstellung. IV Bände. Heidelberg: Winter.

Behaghel, Otto (1930): Zur Stellung des adnominalen Genitivs im Germanischen und Deutschen. Zeitschrift für vergleichende Sprachforschung auf dem Gebiete der Indogermanischen Sprachen 57 (1-2), 43-63, 160.

Blaxter, Tam (2017): Speech in space and time. Contact, change and diffusion in medieval Norway. Dissertation, University of Cambridge.

Carr, Charles T. (1933): The position of the genitive in German. The Modern Language Review 28 (4), 465-479.

Dal, Ingerid (2014): Kurze deutsche Syntax auf historischer Grundlage. Neu bearbeitet von HansWerner Eroms. Berlin, Boston: De Gruyter.

Demske, Ulrike (2001): Merkmale und Relationen. Diachrone Studien zur Nominalphrase des Deutschen. Berlin: De Gruyter.

Donhauser, Karin (1998): Das Genitivproblem und (k)ein Ende? Anmerkungen zur aktuellen Diskussion um die Ursachen des Genitivschwundes. In John Ole Askedal (Hrsg.), Historische germanische und deutsche Syntax. Akten des Internationalen Symposiums anläßlich des 100. Geburtstages von Ingerid Dal, Oslo, 27.9.-1.10.1995, 69-86. Frankfurt a.M. u.a.: Peter Lang.

Durrell, Martin, Paul Bennett, Silke Scheible \& Richard J. Whitt (2012): The GerManC Corpus. Manchester: School of Languages, Linguistics and Cultures.

Ebert, Robert Peter (1988): Variation in the Position of the Attributive Genitive in Sixteenth Century German. Monatshefte 80 (1), 32-49.

Fritze, Marie-Elisabeth (1976): Bezeichnungen für den Zugehörigkeits- und Herkunftsbereich beim substantivischen Attribut. In Gerhard Kettmann \& Joachim Schildt (Hrsg.), Zur Ausbildung der Norm der deutschen Literatursprache auf der syntaktischen Ebene (1470-1730). Der Einfachsatz, 417-476. Berlin: Akademie-Verlag.

Habermann, Mechthild (2012): Leichenpredigten des 17. Jahrhunderts im konfessionellen Kontext. In Jürgen Macha, Anna-Maria Balbach \& Sarah Horstkamp (Hrsg.), Konfession und Sprache in der Frühen Neuzeit. Interdisziplinäre Perspektiven, 63-84. Münster u.a.: Waxmann.

Harczyk, Ignaz (1898): Gotes. Eine Anmerkung zur altdeutschen Wortstellung. Beiträge zur Geschichte der deutschen Sprache und Literatur (PBB) 23, 240-245.

Kettmann, Gerhard \& Joachim Schildt (Hrsg.) (1976): Zur Ausbildung der Norm der deutschen Literatursprache auf der syntaktischen Ebene (1470-1730). Der Einfachsatz. Berlin: Akademie-Verlag.

Kopf, Kristin (2018): Fugenelemente diachron. Eine Korpusuntersuchung zu Entstehung und Ausbreitung der verfugenden $\mathrm{N}+\mathrm{N}$-Komposita. Berlin, Boston: De Gruyter.

Kopf, Kristin (eingereicht): Was ist so besonders an Gott? Ein grammatischer Abweichler im Frühneuhochdeutschen. In Luise Kempf, Damaris Nübling \& Mirjam Schmuck (Hrsg.), Linguistik der Eigennamen. Berlin: De Gruyter.

Lanouette, Ruth Lunt (1998): The Attributive Genitive in Early New High German: A Semantic Analysis. American Journal of Germanic Linguistics and Literature 10 (1), 73-90.

Macha, Jürgen (2014): Der konfessionelle Faktor in der deutschen Sprachgeschichte der Frühen Neuzeit. Würzburg: Ergon. 
Niehaus, Konstantin (2016): Wortstellungsvarianten im Schriftdeutschen. Über Kontinuitäten und Diskontinuitäten in neuhochdeutscher Syntax. Heidelberg: Winter.

Nübling, Damaris, Antje Dammel, Janet Duke \& Renata Szczepaniak (2017): Historische Sprachwissenschaft des Deutschen. Eine Einführung in die Prinzipien des Sprachwandels. 5. Aufl. Tübingen: Narr.

Oubouzar, Erika (1997): Syntax und Semantik des adnominalen Genitivs im Althochdeutschen. In Yvon Desportes (Hrsg.), Semantik der syntaktischen Beziehungen. Akten des Pariser Kolloquiums zur Erforschung des Althochdeutschen 1994, 223-244. Winter: Heidelberg.

Pavlov, Vladimir M. (1983): Zur Ausbildung der Norm der deutschen Literatursprache im Bereich der Wortbildung (1470-1730). Von der Wortgruppe zur substantivischen Zusammensetzung. Berlin: Akademie-Verlag.

Pickl, Simon (2017): Neues zur Entwicklung der Negation im Mittelhochdeutschen. Grammatikalisierung und Variation in oberdeutschen Predigten. Beiträge zur Geschichte der deutschen Sprache und Literatur (PBB) 139 (1), 1-46.

Pickl, Simon (im Erscheinen a): Polarisation and the Emergence of a Written Marker. A Diachronic Corpus Study of the Adnominal Genitive in German. Journal of Germanic Linguistics.

Pickl, Simon (im Erscheinen b): Factors of Selection, Standard Universals, and the Standardisation of German Relativisers. Language Policy (Sonderheft Revisiting Haugen. Alternative Histories of Standardization, hg. von Gijsbert Rutten und Rik Vosters).

Pickl, Simon, Aaron Spettl, Simon Pröll, Stephan Elspaß, Werner König \& Volker Schmidt (2014): Linguistic Distances in Dialectometric Intensity Estimation. Journal of Linguistic Geography 2 (1), 25-40.

Prell, Heinz-Peter (2000): Die Stellung des attributiven Genitivs im Mittelhochdeutschen. Zur Notwendigkeit einer Syntax mittelhochdeutscher Prosa. Beiträge zur Geschichte der deutschen Sprache und Literatur (PBB) 122 (1), 23-39.

Pröll, Simon, Simon Pickl, Aaron Spettl, Volker Schmidt, Evgeny Spodarev, Stephan Elspaß \& Werner König (2015): Neue Dialektometrie mit Methoden der stochastischen Bildanalyse. In Roland Kehrein, Alfred Lameli \& Stefan Rabanus (Hrsg.), Regionale Variation des Deutschen: Projekte und Perspektiven, 173-194. Berlin: De Gruyter Mouton.

Reichmann, Oskar \& Klaus-Peter Wegera (Hrsg.) (1993): Frühneuhochdeutsche Grammatik. Von Robert Peter Ebert, Oskar Reichmann, Hans-Joachim Solms \& Klaus-Peter Wegera. Tübingen: Niemeyer.

Scott, Alan K. (2014): The genitive case in Dutch and German. A study of morphosyntactic change in codified languages. Leiden, Boston: Brill.

Silverstein, Michael (1976): Hierarchy of features and ergativity. In Robert M.W. Dixon (Hrsg.), Grammatical Categories in Australian Languages, 112-171. Canberra: Australian Institute of Aboriginal Studies.

Solling, Daniel (2011): Zu prä- und postnominalen Genitivattributen in frühneuhochdeutschen Predigten. In Elisabeth Wåghäll Nivre, Brigitte Kaute, Bo Andersson, Barbro Landén \& Dessislava Stoeva-Holm (Hrsg.), Begegnungen. Das VIII. Nordisch-Baltische Germanistentreffen in Sigtuna vom 11. bis zum 13. 6. 2009, 269-282. Stockholm: Stockholm University. Tükör, Christina (2008): Genitiv im Frühneuhochdeutschen. Magisterarbeit, Universität Wien. Wagner, Wilhelm (1905): Die Stellung des attributiven Genitivs im Deutschen. Ein Kapitel aus der Lehre von der deutschen Wortstellung, zugleich ein Beitrag zur Entstehungsgeschichte der unechten Komposita. Darmstadt: G. Otto's Hof-Buchdruckerei.

Wandt, Corinna (2012): Die Schreibsprache des Julius Pflug im Konfessionsstreit. Schreibsprachanalyse und ein edierter Dialog. Berlin: Akademie Verlag. 